\title{
1 High mobility group 20B (HMG20B) is a differentially expressed gene in human metastatic breast cancer, in the brain and in the lymph nodes.
}

Shahan Mamoor, MS ${ }^{1}$

1'shahanmamoor@gmail.com

East Islip, NY USA

Metastasis to the brain is a clinical problem in patients with breast cancer ${ }^{1-3}$. We mined published microarray data ${ }^{4,5}$ to compare primary and metastatic tumor transcriptomes for the discovery of genes associated with brain metastasis in humans with metastatic breast cancer. We found that high mobility group 20B, encoded by HMG20B, was among the genes whose expression was most different in the brain and lymph node metastases of patients with metastatic breast cancer. HMG20B mRNA was present at increased quantities in brain metastatic tissues as compared to primary tumors of the breast. Importantly, expression of HMG20B in primary tumors was significantly correlated with patient distant metastasis-free survival in patients with breast cancer. Modulation of HMG20B expression may be relevant to the biology by which tumor cells metastasize from the breast to the brain while evading immune clearance in the lymph nodes in humans with metastatic breast cancer.

Keywords: breast cancer, metastasis, brain metastases, central nervous system metastases, lymph node metastases, high mobility group 20B, HMG20B, systems biology of breast cancer, targeted therapeutics in breast cancer. 
One report described a $34 \%$ incidence of central nervous system metastases in patients compared administration of either neratinib or trastuzumab in conjunction with paclitaxel demonstrated that in a randomized, controlled setting, in breast cancer patients treated with neratinib, not only was the incidence of central nervous system recurrence significantly lower, the time to central nervous system metastasis was significantly delayed as compared to patients administered trastuzumab ${ }^{6}$. The alarmingly high rate of central nervous system metastasis described, as well as data, both anecdotal ${ }^{2}$ and from a randomized, controlled setting ${ }^{6}$ illustrating that treatment with trastuzumab may be associated with these events demands an enhanced understanding of the transcriptional makeup of brain metastatic tissues to support identification of therapeutic targets, whether they are treatment related or not. We performed a global comparative analysis of primary and metastatic tumors in patients with brain metastatic breast cancer ${ }^{4,5}$. We discovered differential and increased expression of the gene encoding high mobility group 20B, HMG20B, in brain metastatic tissues of patients with metastatic breast cancer.

\section{$\underline{\text { Methods }}$}

We used datasets GSE $10893^{4}$ and GSE $124648^{5}$ for this global differential gene expression analysis of brain metastatic breast cancer in conjunction with GEO2R. GSE10893 was generated using Agilent-011521 Human 1A Microarray G4110A technology with $n=11$ primary breast tumors and $n=3$ brain metastases from patients with breast cancer; analysis was performed using platform GPL885. GSE124648 was generated using Affymetrix Human Genome U133A array technology with $n=10$ normal breast tissues and $n=44$ lymph node metastases from patients with breast cancer; analysis was performed using platform GPL96. The Benjamini and Hochberg method of $p$-value adjustment was used for ranking of differential expression but raw $p$-values were used to assess statistical significance of global differential expression. Log-transformation of data was auto-detected, and the NCBI generated category of platform annotation was used. A statistical test was performed to evaluate whether HMG20B gene expression was significantly different between primary tumors of the breast and brain metastases in humans with breast cancer using a two-tailed t-test. For Kaplan-Meier survival analysis, we used the Kaplan-Meier plotter online tool ${ }^{7}$ for correlation of HMG20B mRNA expression levels in primary tumors of the breast with distant metastasis-free survival in $n=1803$ breast cancer patients.

\section{$\underline{\text { Results }}$}

We performed global comparative transcriptome analysis of metastatic and primary tumor tissues of patients with metastatic breast, as well as normal tissues of the breast using published microarray data ${ }^{4,5}$ to describe the transcriptional landscape of brain metastasis in human breast cancer in an unbiased fashion and for the discovery of novel therapeutic targets.

\section{HMG20B is differentially expressed in the brain metastases of patients with brain metastatic breast cancer.}

Through blind, systems-level analysis of published microarray data ${ }^{4}$, we identified high mobility group 20B, encoded by HMG20B, as a differentially expressed gene in the brain 
metastatic tissues of humans with breast cancer (Table 1). When sorting each of the genes expressed in brain metastases based on significance of difference as compared to primary tumors of the breast in patients with breast cancer, HMG20B ranked 18 out of 17418 total transcripts (Chart 1), equating to $99.9 \%$ differential expression. Differential expression of HMG20B in the brain metastases of patients with metastatic breast cancer was statistically significant (Chart 1 ; $p=1.62 \mathrm{E}-05)$.

HMG20B is differentially expressed in the lymph node metastases of patients with breast cancer.

To determine whether differential expression of HMG20B in human breast cancer could be validated in a second microarray dataset, and whether this was specific to brain metastases or could be observed in other anatomical locations, we queried a second microarray dataset ${ }^{5}$, here comparing normal breast tissues to lymph node metastases. Again, we identified HMG20B as a differentially expressed gene in the lymph node metastatic tissues of patients with breast cancer (Chart 2). When sorting each of the genes expressed in lymph node metastases based on significance of difference as compared to normal breast tissues, HMG20B ranked 1345 out of 22283 total transcripts (Chart 2), equating to $94.0 \%$ differential expression. Differential expression of HMG20B in the lymph node metastases of patients with metastatic breast cancer was statistically significant (Chart $2 ; p=6.73 \mathrm{E}-06$ ). Thus, differential expression of HMG20B, transcriptome-wide, in metastatic tissues of women with metastatic breast cancer was conserved across two independent microarray datasets, in the brain and in the lymph nodes

\section{HMG20B is expressed at higher levels in the brain metastases of patients with metastatic} breast cancer.

We obtained exact mRNA expression levels for HMG20B, in primary tumors of the breast and in brain metastasis of patients with brain metastatic breast cancer to determine direction and statistical significance of change in HMG20B expression in brain metastatic tissues. HMG20B was expressed at higher levels in the brain metastases of patients with breast cancer as compared to primary tumors of the breast, and this difference was statistically significant (Figure 1; $p=0.000016)$.

\section{HMG20B expression is significantly correlated with survival outcomes in human breast} cancer.

We performed Kaplan-Meier survival analysis ${ }^{8}$ in 1803 breast cancer patients in total, to evaluate whether HMG20B tumor expression was correlated with survival outcomes in breast cancer. We observed a statistically significant correlation between primary tumor expression of HMG20B and distant metastasis-free survival (DMFS) for patients in the upper survival quartile (Figure 2). Patients whose primary tumors expressed low levels of HMG20B possessed median DMFS of 87.6 months, while patients whose tumors expressed high levels of HMG20B possessed median DMFS of 125.65 months. This difference in DMFS based on HMG20B tumor expression in patients with breast cancer was statistically significant (Figure 2, Chart 3; logrank p-value: 0.035 ; hazard ratio: $0.78(0.62-0.98))$. 
Thus, by mining published microarray data ${ }^{4,5}$ in an unbiased and systematic fashion, we identified high mobility group 20B, encoded by HMG20B, as among the genes whose expression was most different, transcriptome-wide, in the brain and lymph node metastases of patients with breast cancer, when compared to primary tumors of the breast and to normal breast tissues, respectively; we observed significantly increased expression of HMG20B in brain metastases as compared to primary tumors of the breast. Further, we found a significant correlation between HMG20B expression and patient survival outcomes, as distant metastasis-free survival was significantly higher in patients whose primary tumors expressed higher levels of HMG20B as compared to patients whose primary tumors expressed lower levels of HMG20B.

\section{Discussion}

We provided evidence here that high mobility group 20B, encoded by HMG20B, is among the genes whose expression is most different in the brain and lymph node metastases of patients with metastatic breast cancer, that HMG20B mRNA is present at significantly increased quantities in brain metastatic tissues as compared to primary tumors of the breast, and that primary tumor HMG20B expression is significantly correlated with patient survival outcomes in human breast cancer. Evaluation of the effects of genetic depletion of HMG20B in mouse models of metastatic breast cancer on metastasis to the central nervous system is merited. Modulation of HMG20B expression may be relevant to the processes by which breast cancer cells exit the breast, enter the vasculature and/or lymphatics, reside in the lymph nodes, evade immune clearance, breach the blood-brain barrier and colonize the brain. These data are one piece of evidence that allude to a common cell or cell(s) of origin for brain and lymph node metastases in human metastatic breast cancer. 


\section{References}

1. Lin, N.U., Amiri-Kordestani, L., Palmieri, D., Liewehr, D.J. and Steeg, P.S., 2013. CNS metastases in breast cancer: old challenge, new frontiers.

2. Bendell, J.C., Domchek, S.M., Burstein, H.J., Harris, L., Younger, J., Kuter, I., Bunnell, C., Rue, M., Gelman, R. and Winer, E., 2003. Central nervous system metastases in women who receive trastuzumab-based therapy for metastatic breast carcinoma. Cancer, 97(12), pp.2972-2977.

3. Tsukada, Y., Fouad, A., Pickren, J.W. and Lane, W.W., 1983. Central nervous system metastasis from breast carcinoma autopsy study. Cancer, 52(12), pp.2349-2354.

4. Weigman, V.J., Chao, H.H., Shabalin, A.A., He, X., Parker, J.S., Nordgard, S.H., Grushko, T., Huo, D., Nwachukwu, C., Nobel, A. and Kristensen, V.N., 2012. Basal-like Breast cancer DNA copy number losses identify genes involved in genomic instability, response to therapy, and patient survival. Breast cancer research and treatment, 133(3), pp.865-880.

5. Sinn, B.V., Fu, C., Lau, R., Litton, J., Tsai, T.H., Murthy, R., Tam, A., Andreopoulou, E., Gong, Y., Murthy, R. and Gould, R., 2019. SET ER/PR: a robust 18-gene predictor for sensitivity to endocrine therapy for metastatic breast cancer. NPJ breast cancer, 5(1), pp.1-8.

6. Awada, A., Colomer, R., Inoue, K., Bondarenko, I., Badwe, R.A., Demetriou, G., Lee, S.C., Mehta, A.O., Kim, S.B., Bachelot, T. and Goswami, C., 2016. Neratinib plus paclitaxel vs trastuzumab plus paclitaxel in previously untreated metastatic ERBB2-positive breast cancer: the NEfERT-T randomized clinical trial. JAMA oncology, 2(12), pp.1557-1564.

7. Györffy, B., Lanczky, A., Eklund, A.C., Denkert, C., Budczies, J., Li, Q. and Szallasi, Z., 2010. An online survival analysis tool to rapidly assess the effect of 22,277 genes on breast cancer prognosis using microarray data of 1,809 patients. Breast cancer research and treatment, 123(3), pp.725-731. 


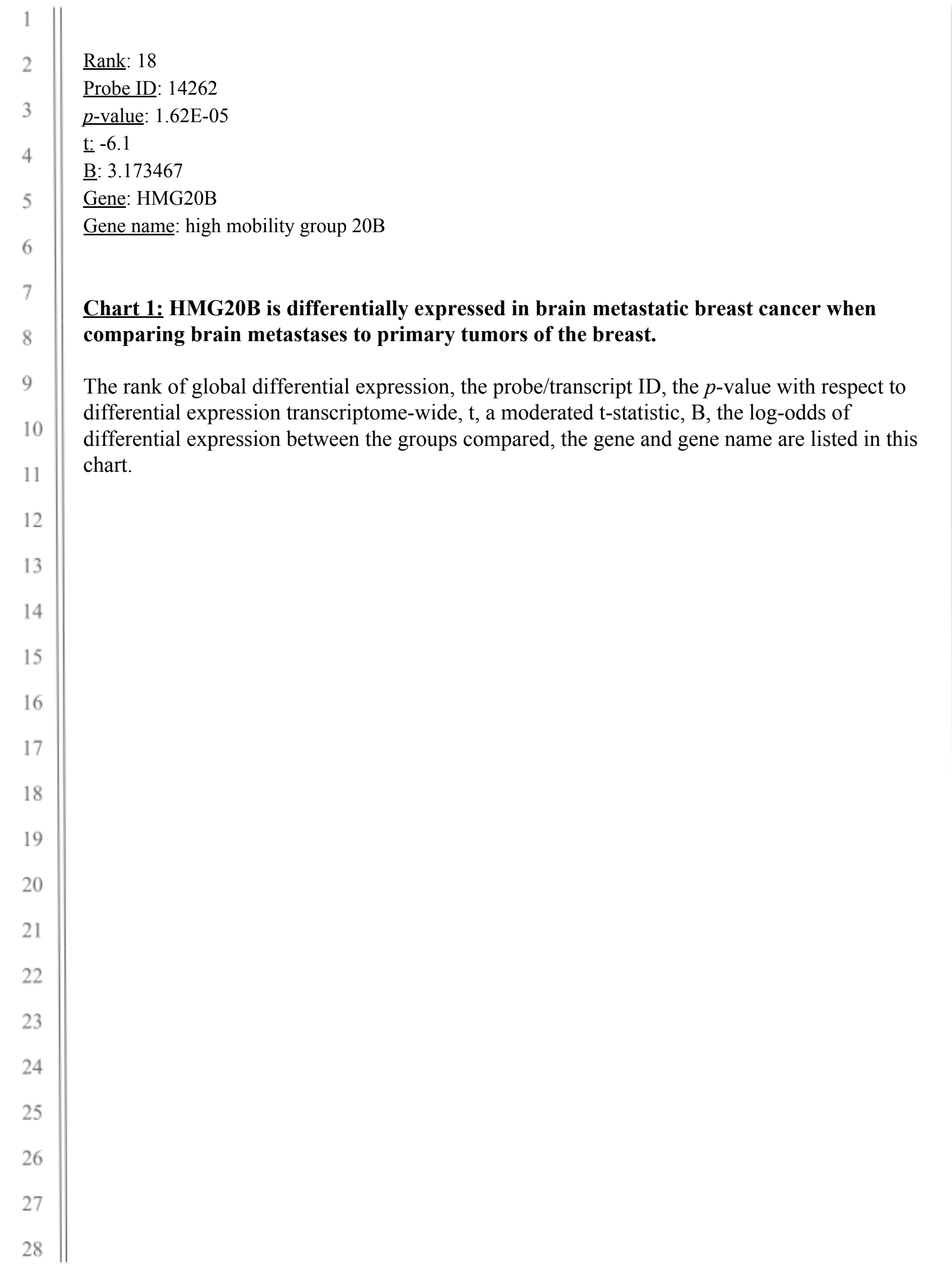




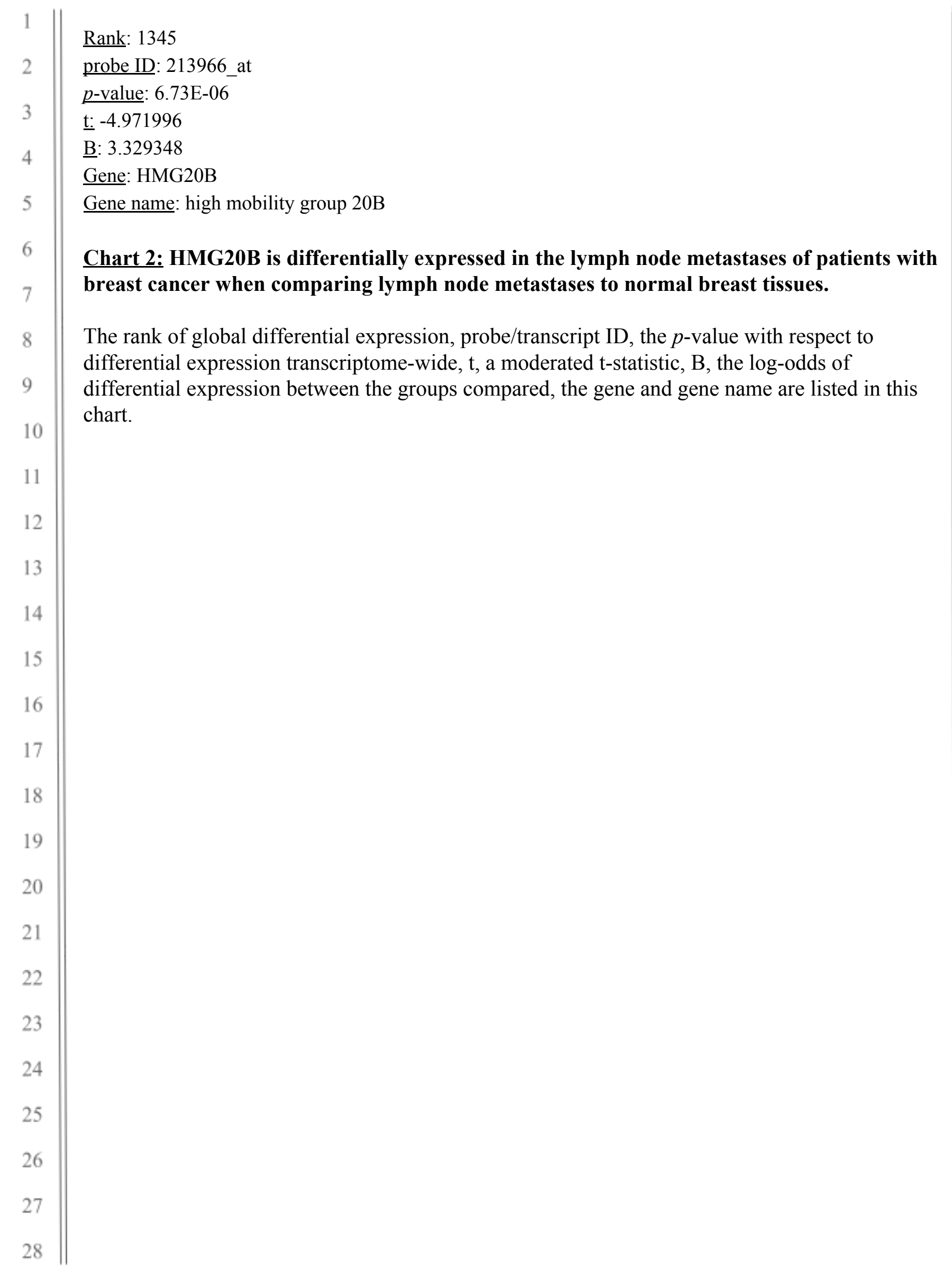




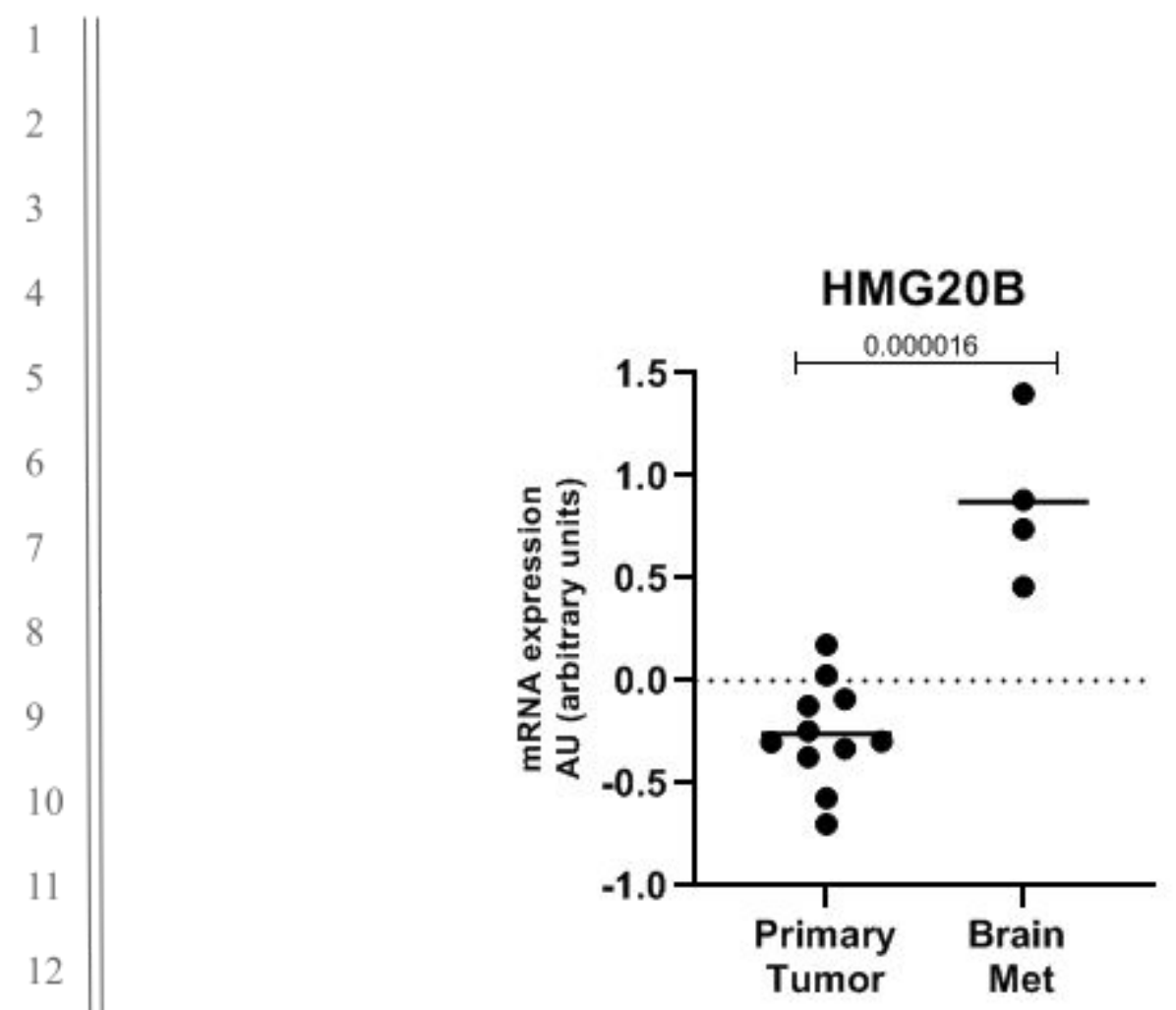

Figure 1: HMG20B is expressed at significantly higher levels in the brain metastases of patients with metastatic breast cancer when compared to primary tumors of the breast.

The mRNA expression level of HMG20B in primary tumors of the breast (left) and in brain metastases of women with metastatic breast cancer (right) is graphically depicted; the result of a statistical test evaluating significance of difference in HMG20B expression between primary tumors and brain metastases is $p=0.000016$. 


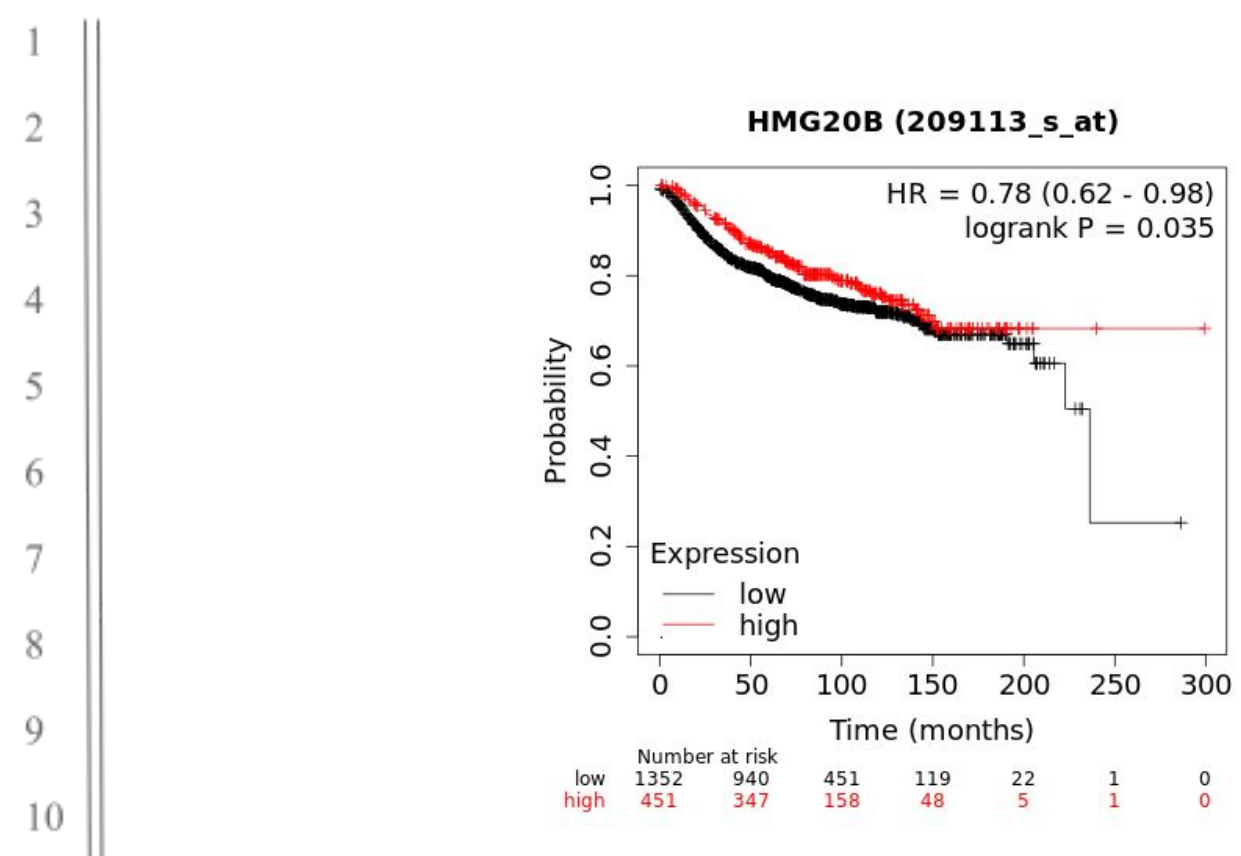

11 Figure 2: Significant correlation between HMG20B primary tumor expression and distant metastasis-free survival in patients with breast cancer.

Depicted in this Kaplan-Meier plot is the probability of distant metastasis-free survival for $n=1803$ total patients stratified into two groups, based on low or high expression of HMG20B in patient primary tumors. The log rank $p$-value denoting statistical significance of difference in distant metastasis-free survival when comparing the two groups, as well as hazard ratio for this comparison is listed above. Listed below is the number of patients at risk (number of patients alive) per interval, after stratification based on HMG20B expression; in the first interval, number at risk is number of patients alive; in each subsequent interval, number at risk is the number at risk less those who have expired or are censored. 


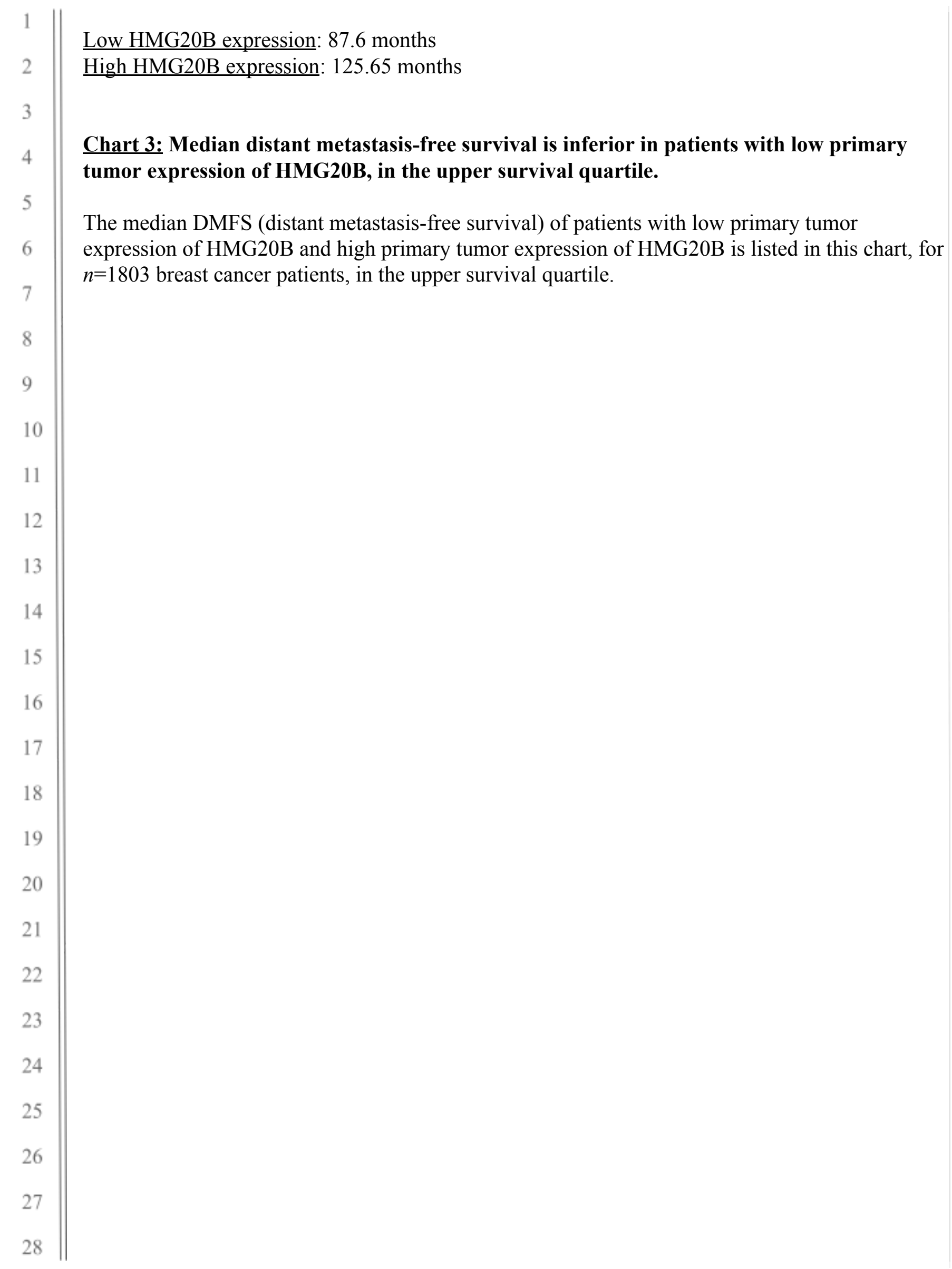

